\title{
Kinetics of Ballistically-Controlled Reactions
}

\author{
E. Ben-Naim, ${ }^{\dagger}$ P. Krapivsky, ${ }^{\dagger}$ F. Leyvraz, ${ }^{\ddagger}$ and S. Redner ${ }^{*}, \dagger$ \\ Center for Polymer Studies and Department of Physics, Boston University, Boston, Massachusetts 02215, and \\ Instituto de Fisica, Laboratorio der Cuernavaca, UNAM, Mexico
}

Received: January 4, 1994; In Final Form: March 25, $1994^{\circ}$

\begin{abstract}
We investigate the kinetics of generic single-species reaction processes when the reactants move ballistically, namely ballistic annihilation, $\mathrm{A}+\mathrm{A} \rightarrow 0$, and a ballistic aggregation process which mimics traffic flow on a single-lane roadway. For ballistic annihilation, dimensional analysis shows that the concentration and root means square velocity decay as $c \sim t^{-\alpha}$ and $v \sim t^{-\beta}$, respectively, with $\alpha+\beta=1$ in any spatial dimension. Analysis of the Boltzmann equation for the evolution of the velocity distribution predicts $\alpha=(2+2 \mu) /(3+$ $2 \mu)$ and $\beta=1 /(3+2 \mu)$ for an initial velocity distribution $P(v, t=0) \sim v^{\mu}$ as $v \rightarrow 0$. New phenomena associated with discrete initial velocity distributions and with mixed ballistic and diffusive reactant motion are also discussed. In the aggregation process, each "car" moves at its initial velocity until the preceding car or cluster is overtaken after which the incident car assumes the velocity of the cluster which it has just joined. For $P_{0}(v) \sim v^{\mu}$ as $v$ $\rightarrow 0$, the average cluster size grows as $n \sim t^{(\mu+1) /(\mu+2)}$ and the average velocity decays as $v \sim t^{-1 /(\mu+2)}$. We also derive an asymptotic expression for the joint distribution function for the cluster mass and velocity.
\end{abstract}

\section{Introduction}

In irreversible diffusion-controlled reactions, it is well-known that the density decays slower than the predictions of mean-field theory in sufficiently low spatial dimension. For example, in single species annihilation, ${ }^{1} \mathrm{~A}+\mathrm{A} \rightarrow 0$, the density decays as $t^{d / 2}$ for spatial dimension $d<2$, while for $d \geq 2$, the density decays according to the mean-field prediction of $t^{-1}$. The contrasting situation where the reactants move ballistically has received much less attention, however, and the scope of established of results is correspondingly sparser. Here, we present several new results for the kinetics of simple reactions when the rate limiting step of the process is the ballistic transport of reactants. Such ballistically-controlled reactions appear simpler than their diffusion-controlled counterparts, since the only stochasticity is the initial velocity distribution of the reactants. However, unanticipated phenomena occur which depend on the form of the initial velocity distribution.

Two general results have been established for ballistic reactions. Elskens and Frisch ${ }^{2}$ introduced single species annihilation, A + $\mathrm{A} \rightarrow 0$ in one dimension, where each particle velocity is $\pm v_{0}$; i.e., the initial velocity distribution $P(v, t=0)=p \delta\left(v-v_{0}\right)+q \delta(v+$ $v_{0}$ ), with $q=1-p$. From probablistic arguments, the density was found to decay as

$$
c(t) \propto(c(0) / v t)^{1 / 2}
$$

in the interesting case of $p=q=1 / 2$. Elskens and Frisch also argued that this result can be understood qualitatively by considering density fluctuations in a linear domain of length $l$ where there will typically be an imbalance in the number of rightmoving and left-moving particles of magnitude $\delta n \simeq(c(0) l)^{1 / 2}$. After a time $t=l / v$, only this residual fluctuation will remain inside the domain. Thus the concentration will be of order $c(t)$ $\simeq \delta n / l$. By expressing $l$ in terms of $t$, eq 1 follows.

In a complementary direction, Carnevale et al ${ }^{3}$ and Jiang and Leyvraz ${ }^{4}$ investigated irreversible aggregation, $A_{i}+A_{j} \rightarrow A_{i+j}$, with ballistic trajectories for the aggregates and with momentum conserving collisions. Here the subscript refers to the (conserved) mass of the aggregates. From a scaling approach, Carnevale $e t$ al. showed that the cluster concentration $c(t)$ decayed as

\footnotetext{
$\uparrow$ Boston University.

Instituto de Fisica.

- Abstract published in Advance ACS Abstracts, June 15, 1994.
}

$$
c(t) \sim t^{-\alpha}, \text { with } \alpha=2 d /(d+2)
$$

It is instructive to reproduce this result by a mean-free-path argument. For simplicity, consider a monomer-only initial condition in which each particle has the same speed but with a random direction. Consequently the momentum of an aggregate of mass $m$ is proportional to $m^{1 / 2}$, since it is the sum of $m$ random momenta, and the corresponding aggregate velocity is $v=p / m$ $\propto m^{-1 / 2}$. From kinetic theory, the time between collisions at any stage of the reaction is $\tau \simeq 1 /(c v \sigma)$, where $c$ is the concentration, $v$ is the typical velocity, and $\sigma$ is the cross-section. In this time interval, each aggregate typically undergoes a collision, leading to a change in concentration $\delta c \sim-c$. Thus

$$
\frac{\mathrm{d} c}{\mathrm{~d} t} \propto \frac{\delta c}{\tau} \simeq-\frac{c}{1 /(c v \sigma)}
$$

For a typical aggregate mass is $m \propto 1 / c$, the right-hand side can be written in terms of the concentration only, using $v \sim c^{-1 / 2}$ and $\sigma \sim c^{-(d-1) / d}$, from which eq 2 follows.

Our investigations are motivated by the above interesting results. In the next section, we consider ballistic single-species annihilation, $\mathbf{A}+\mathbf{A} \rightarrow 0$, with arbitrary initial velocity distributions. We have previously found ${ }^{5}$ that the exponent $\alpha$ depends on the form of the initial velocity distribution and on $d$, while radically different behavior occurs for discrete initial velocity distributions. We will also discuss intriguing results for the case where there is superimposed diffusion on the primary ballistic motion. In the following section, we investigate a ballistic aggregation process which is an idealized description of traffic flow on a one-dimensional roadway. ${ }^{6}$ Our modeling is complementary to traditional continuum descriptions of traffic flows.? In our model, "cars" move ballistically in one direction (to the right) according to an initial velocity distribution. Clusters form whenever a faster car overtakes a slower car or cluster and is then obliged to assume the velocity of the lead car in the cluster. For an initial velocity distribution that varies as $v^{\mu}$ as $v \rightarrow 0$, we determine the average size and velocity of clusters, as well as the form of the joint velocity and mass distribution.

\section{Ballistic Single Species Annihilation}

(a) Continuous Velocity Distributions. Consider a system of identical particles with a zero mean initial velocity distribution, 
$P(v, t=0)$. (For simplicity, we consider a random initial spatial distribution.) Particles move at their initial velocity until a collision occurs, which results in the annihilation of both particles. We wish to determine the time dependence of the concentration, $c(t)=\int \mathrm{d} v P(v, t) \sim t^{-\alpha}$, and the root mean square (rms) (or typical) velocity, $v_{\mathrm{rms}}=\left(\int \mathrm{d} v v^{2} P(v, t) / c(t)\right)^{1 / 2} \sim t^{-B}$.

From mean-free path considerations, the time between collisions for this system with particles of radius $r$ and typical velocity $v_{\text {rms }}$ at concentration $c$ is $t \sim 1 / c v_{\mathrm{rms}} r^{d-1}$, or $c v_{\mathrm{rms}} \propto t^{-1}$. Thus the relation $\alpha+\beta=1$, which should hold for all spatial dimension $d$, follows. Since the lifetime of particles with velocity $v$ is proportional to $1 / v$, faster particles tend to annihilate more quickly, and the typical velocity should decay in time. Consequently $\alpha$ should typically be less than unity, and as shown below, its value depends on the form of the initial velocity distribution.

To determine the evolution of the velocity distribution in one dimension, we analyze the Boltzmann equation. Let $P(x, v, t)$ be the density of particles with velocity $v$ at position $x$ and at time $t$. At time $t+\Delta t$, the velocity distribution changes both because particles move and because of reactions. We treat the reaction term in a mean-field approximation by assuming that a particle of $x^{\prime}\left\langle x\right.$ and velocity $v^{\prime}>v$ necessarily reacts with the target particle at $(x, v)$ when $x-x^{\prime}<\left(v^{\prime}-v\right) \Delta t$. There is a complementary contribution due to collisions between the target and a particle located at $x^{\prime}>x$ with $v^{\prime}<v$. These two contributions lead to

$$
\begin{aligned}
& P(x+v \delta t, v, t+\Delta t)-P(x, v, t)= \\
& -k P(x, v, t)\left[\int_{v}^{\infty} \mathrm{d} v^{\prime} \int_{x-\left(v^{\prime}-v\right) \Delta t}^{x} \mathrm{~d} x^{\prime} P\left(x^{\prime}, v^{\prime}, t\right)+\right. \\
& \left.\quad \int_{-\infty}^{v} \mathrm{~d} v^{\prime} \int_{x}^{x+(v-v) \Delta t} \mathrm{~d} x^{\prime} P\left(x^{\prime}, v^{\prime}, t\right)\right]
\end{aligned}
$$

where $k$ is a dimensionless reaction constant. This approximate equation overcounts collisions, since the incident particle at $x^{\prime}$ may react with a third particle rather than with the target particle. We assume that the contributions of these three-body effects are negligible.

This Boltzmann equation can be simplified by expanding the first order in $\Delta t$ and also assuming spatial homogeneity, so that the spatial derivative can be set to zero. This yields, for the concentration of particles with velocity $v$

$$
\frac{\partial P(v, t)}{\partial t}=-k P(v, t) \int_{-\infty}^{\infty} \mathrm{d} v^{\prime} \mid v-v 1 P\left(v^{\prime}, t\right)
$$

where the $|v-v|$ dependence of the integral kernel controls the reaction rate. Although there are uncontrolled approximations underlying eq 5 , this formulation gives a useful quantitative description of the decay kinetics.

This integral equation can be analyzed by scaling. We assume that the velocity distribution has the scaled form

$$
P(v, t)=\frac{c_{0}}{v_{0}}\left(\frac{t}{t_{0}}\right)^{\beta-\alpha} f(z), \text { with } z=\frac{v}{v_{0}}\left(\frac{t}{t_{0}}\right)^{\beta}
$$

where $z$ is the dimensionless velocity, $t_{0}=1 /\left(k c_{0} v_{0}\right)$ the initial time between reactions, $k$ the dimensionless reaction constant, $c_{0}$ the initial concentration, and $v_{0}$ an initial typical velocity. Substituting this scaling form into eq 5 , the exponent relation $\alpha$ $+\beta=1$ follows. Additionally, we obtain an equation for the scaling function

$$
(2 \beta-1) f(z)+\beta z f^{\prime}(z)=-f(z) \int_{-\infty}^{\infty} \mathrm{d} z^{\prime}|z-z| f\left(z^{\prime}\right)
$$

By examining separately the large- $z$ and small- $z$ behavior of this equation, one can verify that an initial velocity distribution with a power-law tail at small velocities leads to a scaling form for the time-dependent velocity distribution. Namely, if the initial velocity distribution has the form $P(v, t=0) \propto|v| \mu \theta\left(v_{0}-|v|\right)$, where $\theta$ is the Heaviside step function, then it is possible to derive that $f(z)$ has the asymptotic forms $f(z) \sim z^{\mu}$ as $z \rightarrow 0$ and $f(z) \sim e^{-k / / \beta}$ as $z \rightarrow \infty$. If we then make that ansatz that the full scaling function has the product form $|z| \mu-|-| x \mid / \beta$, we then find $\beta=1 /(3+$ $2 \mu) .^{5}$

Thus a scaling analysis of the Boltzmann equation predicts that $\alpha$ and $\beta$ can take on any value between 0 and 1 as $\mu$ is varied, subject to the condition $\alpha+\beta=1$. Notice that when the concentration decays relatively quickly, $\alpha \cong 1$, the typical velocity decays slowly, and vice versa. The generalization of this scaling analysis to higher spatial dimensions yields $\beta=1 /(1+2 d+2 \mu)$. The agreement between the numerical integration of the Boltzmann equation, eq 5 , and Monte Carlo simulations is quite good. Notice that as the spatial dimension increases, the limiting value $\alpha=1$, corresponding to the transparent limit, $\dot{c}=-k c^{2}$, is approached but never reached. Only in the $d=\infty$ limit are particle trajectories sufficiently independent that the typical velocity does not decrease. This is in contrast to many diffusion-controlled reactions for which transparent behavior occurs when $d \geq d_{c}$ with $d_{c}$ finite. ${ }^{1}$

(b) Discrete Velocity Distributions. Unusual and thus far unexplained features occur for discrete initial velocity distributions. In this case, the Boltzmann equation discussed above reduces to a finite set of (mean-field) rate equations. For many situations of interest, these equations can be solved in closed form. However, while the Boltzmann equation solutions and simulation results in one dimension are in good agreement for continuous velocity distributions, there are basic discrepancies in the corresponding results for discrete distributions. The apparent success of the Boltzmann approach for continuous distributions stems from the fact that the velocities of nearby particles remain nearly uncorrelated at long times. In contrast, for discrete velocity distributions, long-range velocity correlations build up as the reaction proceeds. Thus it is to be anticipated that the rate equations approach may not accurately describe the asymptotic behavior of one-dimensional systems with discrete initial velocity distributions.

To appreciate this situation in a generic system with a discrete velocity distribution, consider, for example, the trimodal case where $P(v, t=0)=p_{+} \delta(v-1)+p_{0} \delta(v)+p_{-} \delta(v+1)$, with $p_{+}+p_{0}$ $+p_{-}=1$. For the symmetric case of $p_{+}=p_{-}$, the Boltzmann equation for the concentrations of the stationary and mobile species, $c_{0}(t)$ and $c_{ \pm}(t)$, respectively, reduces to the rate equations

$$
\begin{aligned}
& \dot{c}_{0}=-2 c_{0} c_{ \pm} \\
& \dot{c}_{ \pm}=-c_{0} c_{ \pm}-2 c_{ \pm}{ }^{2}
\end{aligned}
$$

The numerical factors of 2 are critical in the qualitative results. While the exact solution can be written in terms of the exponential integral, it is more instructive to write only the asymptotic behaviors

$$
\begin{aligned}
c_{ \pm}(t) & \sim \frac{1}{2} c_{0}(\infty) e^{-c_{0}(\infty) t} \\
c_{0}(t) & \sim c_{0}(\infty) \exp \left[e^{-c_{0}(\infty) t}\right]
\end{aligned}
$$

where $c_{0}(\infty)=c_{0}(t=0) e^{-2 c_{ \pm}(0) / c_{0}(0)}$. According to the rate equations, the concentration of mobile particles decays exponentially in time, while a residue of stationary particles always remains whose concentration is vanishingly small if the initial concentration is relatively small.

Numerical simulations of this process indicate a rather different behavior. Because of subtle crossover effects, a direct simulation turns out to be inadequate to yield accurate results. We therefore developed an alternative approach in which all collision partners and the corresponding collision times are identified at the outset. With this method we can simulate $5 \times 10^{5}$ particles to $10^{5}$ time 
steps in of the order of 30 cpu seconds on a DEC/AXP 3000/400 workstation. These simulations reveal the following: For $c_{0}(0)$ $<1 / 4, c_{0}(t) \sim 1 / t$, and $c_{ \pm}(t) \sim t^{-1 / 2}$, but with the asymptotic behavior setting in more slowly as $c_{0}(0)$ is increased. (This slow crossover was erroneously reported as nonuniversal behavior in ref 5.) When $c_{0}(0)=1 / 4, c_{0}(t)=c_{ \pm}(t) \sim t^{-2 / 3}$. For $c_{0}(0)>1 / 4$, $c_{0}(t)$ saturates to a finite limiting value which appears to be proportional to $\left(c_{0}(0)-(1 / 4)^{2}\right.$, while $c_{ \pm}(t)$ decays faster than a power law.

It is worth noting that the two different exponent values for the case $c_{0}(0)<1 / 4$ can be understood in the limit of $c_{0}(0) \rightarrow$ 0 . In this limit, $c_{ \pm}(t) \sim t^{-1 / 2}$, because the system reduces to the Elskens-Frisch model. On the other hand, a stationary particle survives only if it is not annihilated by particles incident from either direction. Since the probabilities of each of these two events is independent, it follows that $c_{0}(t) \sim c_{ \pm}(t)^{2} \sim t^{-1}$. However, a similar qualitative argument has not yet been found for the other qualitative behaviors in the trimodal system.

(c) Mixed Ballistic and Diffusive Motion. For a discrete initial velocity distribution, particles with the same velocity never meet. However, a superimposed diffusion provides a mechanism for same-velocity particles to annihilate, a feature which leads to interesting behavior. Thus consider the Elskens and Frisch bimodal velocity model, ${ }^{2}$ in which each particle undergoes superimposed diffusion, with diffusion coefficient $D$, in addition to the $\pm v_{0}$ ballistic motion. The resulting kinetics can be treated by dimensional analysis. From the parameters of the system, the initial concentration $c_{0}, v_{0}$, and $D$, the only variable combinations with the dimensions of concentration are, $c_{0}, 1 / v_{0} t$, and $1 /(D t)^{1 / 2}$. Accordingly, the time-dependent concentration must have the form

$$
c(t) \propto\left(c_{0}\right)^{\mu}\left(\frac{1}{v_{0} t}\right)^{v}\left(\frac{1}{(D t)^{1 / 2}}\right)^{1-\mu-v}
$$

We determine $\mu$ and $v$ by requiring that $c(t)$ matches with the known limiting behaviors in the cases of negligible drift ${ }^{1}$ and negligible diffusion ${ }^{2}$ at the appropriate crossover times. In the former case eq 10 should reduce to the diffusion-limited result $c(t) \rightarrow(D t)^{-1 / 2}$ when $t<\tau_{v} \simeq D / v_{0}^{2}$, the crossover time below which drift effects can be ignored. Conversely, in the limit of small $D$, eq 10 should reduce to $c(t) \rightarrow\left(c_{0} / v_{0} t\right)^{1 / 2}$ for $t<\tau_{d} \simeq$ $1 /\left(D c_{0}^{2}\right)$, which is the time for adjacent particles to meet by diffusion. By matching the general result eq 10 to the limiting behaviors at the appropriate crossover times, we find

$$
c(t) \sim\left(\frac{1}{v_{0} t}\right)^{1 / 2}\left(\frac{1}{(D t)^{1 / 2}}\right)^{1 / 2} \propto t^{-3 / 4}
$$

Interestingly, the density decays as $t^{-1 / 2}$ for both the diffusionlimited and the ballistically-limited reactions, but if both effects occur the density decays as $t^{-3 / 4}$.

\section{Aggregation Model for One-Dimensional Traffic Flow}

Our traffic flow model is (unidirectional) ballistic aggregation in which the product takes on the velocity of the slower of the two incident particles, i.e.

$$
\mathbf{A}_{n_{1}, v_{1}}+\mathbf{A}_{n_{2}, v_{2}} \rightarrow \mathbf{A}_{n_{1}+n_{2}, \min \left\{v_{1}, v_{2}\right\}}
$$

where $A_{n, v_{l}}$ denotes of cluster with $n_{l}$ cars and velocity $v_{i}$. We first apply scaling, together with the statistical properties of the minimal random variable in a large sample, to determine the decay of the velocity and the cluster concentration. We then solve for the distribution of cluster velocities by consideration of a reduced problem where the cluster mass is ignored. Finally, we give the asymptotic distribution for the joint mass and velocity distribution. (a) Scaling Analysis. Consider the initial condition of isolated cars ("monomers") which are randomly distributed on the line at time $t=0$, and initial velocity distributions which can be written in the scaling form

$$
P(v, t=0)=\frac{c_{0}}{v_{0}} P_{0}\left(\frac{v}{v_{0}}\right) \quad v>0
$$

with $\int_{0}{ }^{\infty} P_{0}(z) \mathrm{d} z=1$. Here we subtract the finite value of the velocity of the slowest car from all velocities. We also consider zero-size cars, appropriate for describing the low-density limit.

Let $m$ and $v$ be the typical cluster mass (equivalently, the number of cars in the cluster) and cluster velocity at time $t$. Thus, the typical distance, $l$, between clusters grows with time as $l \sim$ $v t$. Since the typical number of particles in a cluster is proportional to the typical distance between clusters, one has $m \sim l \sim v t$. To find the typical velocity, one has to relate the size of a cluster to its velocity. Such a relation may be found exactly for an auxiliary "one-sided" problem in which particles are placed with a fixed density $c_{0}$ to the left of a given particle and no particles are placed to the right. Eventually, this particle moving with velocity $v$ will form a cluster that includes all consecutive particles to its left whose initial velocities larger than $v$. The probability that there are exactly $k$ such particles is equal to $P_{-} P_{+}{ }^{k}$, with $P_{+}(v)\left(P_{-}(v)\right)$ defined as the probability that a particle has velocity larger (smaller) than $v$, i.e., $P_{+}(v)=\int_{v}{ }^{\infty} P_{0}(v) \mathrm{d} v^{\prime}$. Therefore the average number of particles in the cluster that ultimately forms is given by

$$
\langle m(v)\rangle=\sum_{k=1}^{\infty} k P_{-} P_{+}{ }^{k}=P_{+} / P_{-}
$$

For a power-law behavior of the initial velocity distribution for small velocities, $P_{0}(v) \propto v^{\mu}$ for $v \ll 1$ (with $\mu>-1$ for normalizability), we find

$$
\langle m(v)\rangle=\frac{P_{+}(v)}{P_{-}(v)} \cong \frac{1}{v^{\mu+1}}
$$

for sufficiently low velocities. For a particle moving with the typical velocity, it is reasonable to expect that this "one-sided" result gives a correct estimate for the original "two-sided" problem. Combining eq 15 with our previous estimate $m \sim v t$ yields the following asymptotic relations

$$
\begin{gathered}
m \sim c^{-1} \sim t^{\alpha}, \text { with } \alpha=\frac{\mu+1}{\mu+2} \\
v \sim t^{-\beta}, \text { with } \beta=\frac{1}{\mu+2}
\end{gathered}
$$

This qualitative dependence of $\alpha$ and $\beta$ on the form of the initial velocity distribution is reminiscent of the behavior in ballistic annihilation. ${ }^{5}$ In both processes, the decay exponents obey $\alpha+$ $\beta=1$ as a consequence of the relation $c \sim 1 / v t$. However the actual values of the decay exponents are different for the two processes. For example, for a flat distribution of initial velocities (corresponding to $\mu=0$ ), one obtains $\alpha=1 / 2$ for the traffic model while $\alpha \cong 0.76$ is found in simulations of the annihilation process.

Since both the mean size and velocity scale as power laws in time, the joint mass-velocity distribution $P_{m}(v, t)$ is expected to evolve toward a scaling distribution. Taking into account mass conservation, $\int \mathrm{d} v \sum_{m} m P_{m}(v, t)=$ const, we postulate the scaling form

$$
P_{m}(v, t) \cong t^{\beta-2 \alpha} \Phi(M, V)
$$


for the joint distribution, with the scaled mass, $M$, and scaled velocity, $V$, defined by $M=m / t^{\alpha}$ and $V=v t^{\beta}$.

Once the joint mass-velocity distribution function is found, the single variable mass and velocity distributions can be obtained by suitable integrations over the subsidiary variable. Thus we write the velocity distribution, $P(v, t)=\sum_{m} P_{m}(v, t)$, in its scaling form

$$
P(v, t) \simeq t^{\beta-\alpha} \Phi_{v}(V)
$$

with $\Phi_{v}(V)=\int_{0}{ }^{\infty} \mathrm{d} M \Phi(M, V)$, while the scaling form for the cluster-mass distribution, $P_{m}(t)=\int \mathrm{d} v P_{m}(v, t)$, is expected to be

$$
P_{m}(t) \simeq t^{-2 \alpha} \Phi_{m}(M)
$$

with $\Phi_{m}(M)=\int_{0}^{\infty} \mathrm{d} V \Phi(M, V)$.

(b) The Car Survival Probability. To solve the traffic model, consider first the survival probability of a given car. This is equivalent to ignoring the masses of each cluster and focusing only on the velocity distribution function $P(v, t)$. The evolution of this distribution is governed by the "derived" coalescence process

$$
\mathrm{A}_{v_{1}}+\mathrm{A}_{v_{2}} \rightarrow \mathrm{A}_{\left.\min \mid v_{1}, v_{2}\right\}}
$$

The density of particles with velocity $v$ in this coalescence process is then identical to $P(v, t)$, the cluster velocity distribution in the traffic aggregation model.

Let $S(v, t)$ be the survival probability of particles of velocity $v$ at time $t$. Here "survival" means that a car does not overtake any traffic, but an overtaken car is still defined as surviving. The survival probability can be found by considering the possible collisions of a particle with initial velocity $v$ and initial position $x$ with slower particles initially placed to its right. A collision with slower $v^{\prime}$-particles does not occur up to time $t$ if the interval $[x, x+(v-v) t]$ does not include the $v^{\prime}$-particle. Therefore for a continuous initial velocity distribution, $P_{0}(v)$, and a Poissonian initial spatial distribution, the probability that the $v^{\prime}$-particle is not in the interval $\left[x, x+\left(v-v^{\prime}\right) t\right]$ is $e^{\left(-d v^{\prime} P_{0}(v)\left(v-v^{\prime}\right) t\right)}$.

To ensure that a particle will survive up to time $t$, one should account for these pair survival probabilities for every $v^{\prime}<v$. One subtle point in this reasoning is that collisions between a $v^{\prime}$-particle and other particles can be ignored. Namely, if a $v$-particle does not overtake a $v^{\prime}$-particle, then overtaking will still not occur even if the $v^{\prime}$-particle overtakes a still slower $v^{\prime \prime}$-particle. Hence, the survival probability is simply a product of exponential factors for all $v^{\prime}$, with $v^{\prime}<v$. Evaluating this product gives the survival probability

$$
S(v, t)=\exp \left[-t \int_{0}^{v} \mathrm{~d} v^{\prime}\left(v-v^{\prime}\right) P_{0}\left(v^{\prime}\right)\right]
$$

from which the velocity distribution is $P(v, t)=P_{0}(v) S(v, t)$.

For $P_{0}(v) \sim v^{\mu}$ as $v \rightarrow 0$, the asymptotic velocity distribution reaches a form that is independent of the details of the largevelocity tail of the initial distribution

$$
P(v, t) \propto v^{\mu} \exp \left[- \text { const } \times t v^{\mu+2}\right]
$$

This form validates the scaling assumption that the asymptotic decay and the shape of the limiting distribution are determined solely by the exponent $\mu$ which characterizes the low-velocity tail of $P_{0}(v)$. From the above velocity distribution, the total concentration, $c(t)=\int 0_{0}{ }^{\infty} \mathrm{d} v P(v, t)$, and the average cluster velocity $\langle v(t)\rangle=\int \mathrm{d} v v P(v, t) / \int \mathrm{d} v P(v, t)$ are found to agree with eq 16.

(c) The Joint Mass and Velocity Distribution. We can now determine the density of clusters of mass $m$ and velocity $v, P_{m}(v, t)$, for the general traffic model. Consider the auxiliary quantity $Q_{m}(v, t)$, the distribution of clusters of velocity $v$, and mass greater than or equal to $m$. Once this is known, $P_{m}(v, t)$ can be obtained by $P_{m}(v, t)=Q_{m}(v, t)-Q_{m+1}(v, t)$. Consider a cluster of velocity $v$ which contains at least $m$ particles. Number the consecutive particles in a cluster from right to left by the index $i$ and denote the rightmost particle as $i=0$. Denote the initial distance between the $i$ th and $(i-1)$ th particle as $x_{i}$. We first solve for $Q_{2}(v, t)$ and then generalize to any $m$. Since $Q_{2}(v, t)$ is the probability that a cluster of velocity $v$ has at least two particles at time $t$, it is equal to the product of the probability that the particle $i=0$ has survived up to time $t, P(v, t)$, and the probability that the cluster $i=1$ (whose mass may be larger than unity) collides with the particle $i=0$ prior to time $t$.

To calculate this collision probability, note that it is necessary that the collision partner from the left $(i=1)$ has a velocity larger than $v$ and that the distance $x_{1}$ is smaller than $\left(v_{1}-v\right) t$. Since the initial density of particles of velocity $v_{1}$ is $P_{0}\left(v_{1}\right)$, while the probability that an interval of length $x_{1}$ is empty is exp $\left(-x_{1}\right)$, the joint velocity-distance density function for the particle $i=1$ is the product $P_{0}\left(v_{1}\right) \exp \left(-x_{1}\right)$. Integration of this probability density over velocities larger than $v$ and over the proper distance interval gives the collision probability

$$
Q_{2}(t)=P(v, t) \int_{0}^{\infty} \mathrm{d} v_{1} P_{0}\left(v_{1}\right) \int_{x_{1}<\left(v_{1}-v\right) t} \mathrm{~d} x_{1} \exp \left(-x_{1}\right)
$$

To derive the general expression for $Q_{m}(v, t)$, the same joint velocity distance distributions $P_{0}\left(v_{i}\right) \exp \left(-x_{i}\right)$ are integrated over the position and velocity of the $i$ th particle for $i=1, \ldots, m-1$. All $m-1$ particles have to move faster than the lead particle, and to ensure a collision it is required that the distance of the $i$ th particle from the lead obeys $x_{1}+\ldots+x_{i} \leq\left(v_{i}-v\right) t$. Imposing these constraints upon the integration over the velocity and distance of the $m-1$ additional particles yields a cumbersome exact formal expression for the cumulative mass-velocity distribution. ${ }^{6}$ For the case where the initial velocity distribution has the small velocity power-law tail $v^{\mu}$, the asymptotic form of the joint mass-velocity distribution, $P_{m}(v, t)=Q_{m}(v, t)-Q_{m+1}(v, t)$ $\cong-\partial Q_{m} / \partial m$, is

$$
P_{m}(v, t) \propto t^{\beta-2 \alpha} V^{\mu}(V+M)^{\mu+1} \exp \left[- \text { const } \times(V+M)^{\mu+2}\right]
$$

when written in the scaling variables $M=m / t^{\alpha}$ and $V=v t^{\beta}$. Notice that for $\mu=0$, the integration of the joint distribution over all velocity gives the mass distribution $P_{m}(t) \sim t^{-1} \exp \left(-M^{2} / 2\right)$ while the density of fixed-sized clusters $P_{m}(t)=\int_{0}^{\infty} \mathrm{d} v P_{m}(v, t)$, decays as $t^{-2 \alpha}$ in the long-time limit.

\section{Summary}

Ballistically-controlled reactions exhibit a rich variety of decay kinetics which depend on the form of the initial velocity distribution. For ballistic annihilation, $\mathbf{A}+\mathbf{A} \rightarrow 0$, analysis of the Boltzmann equation indicates that the time dependence of the concentration and the typical velocity are governed by exponents whose value depends on the small-velocity limit of the initial velocity distribution. Intriguingly, an initial velocity distribution with a large component of slower particles gives a weak decay of the concentration and relatively faster decay of the typical velocity. As the spatial dimension is increased, the "transparent" limit $\alpha=1$ is approached but apparently never reached. While the Boltzmann equation provides a good phenomenological description of the kinetics, there is, as yet, no microscopic theory. Such a theory might help understand the unusual behavior exhibited by ballistic annihilation with discrete initial velocity distributions and with combined ballistic and diffusive motion.

We also considered a ballistic aggregation model that mimics the kinetics of clustering in a single lane of traffic. By a probabilistic approach, the analytical forms of the cluster velocity distribution and the joint mass-velocity distribution have been 
derived. For an initial velocity distribution with a power-law small-velocity limit, both the average velocity and the average mass of clusters vary algebraically in time with exponents that depend on the small-velocity tail of the initial velocity distribution. The qualitative asymptotic behavior is similar to that observed in the related ballistic annihilation model.

The traffic model has the unrealistic feature of irreversibility, due to the lack of a passing mechanism. This leads to pathological traffic jams in which clusters of cars grow without bound. More realistically, some form of passing needs to be incorporated. For example, once cars are in a cluster, a faster car should be allowed to pass the slower car directly ahead at a rate which is some function of the velocity difference of the two cars. This would allow a fast car to traverse a cluster car-by-car and ultimately regain its intrinsic velocity once the cluster is completely passed. It may prove interesting to examine the steady-state transport properties for this class of models.

Acknowledgment. We thank Ganpathy Murthy and Chris Myers for useful discussions. We also gratefully acknowledge
ARO Grant DAAH04-93-G-0021, NSF Grants INT-8815438 and DMR-9219845, and the donors of The Petroleum Research Fund, administered by the American Chemical Society, for partial support of this research.

\section{References and Notes}

(1) See, e.g., Toussaint, D.; Wilczek, F. J. Chem. Phys. 1983, 78, 2642. Kopelman, R. Science 1988, 241, 1620.

(2) Elskens, Y.; Frisch, H. L. Phys. Rev. A 1985, 31, 3812.

(3) Carnevale, G. F.; Pomeau, Y.; Young, W. R. Phys. Rev. Lett. 1990, $64,2913$.

(4) Jiang, Y.; Leyvraz, F. J. Phys. A 1993, 26, L179.

(5) Ben-Naim, E.; Redner, S.; Leyvraz, F. Phys. Rev. Lett. 1993, 70, 1890.

(6) Ben-Naim, E.; Krapivsky, P.; Redner, S. Phys. Rev. E., in press.

(7) See, e.g., Prigogine, I.; Herman, R. Kinetic Theory of Vehicular Traffic; Elsevier: New York, 1971. Leutzbach, W. Introduction to the Theory of Traffic Flow; Springer-Verlag: Berlin, 1988. Kerner, B. S.; Konhauser, R. Phys. Rev. E 1993, 48, 2335. 